This is an electronic reprint of the original article. This reprint may differ from the original in pagination and typographic detail.

Author(s): Petrov, Dmitry; Melnik, Sergei; Hämäläinen, Timo

Title: $\quad$ Distributed GNSS-Based Time Synchronization and Applications

Year: $\quad 2016$

Version:

Please cite the original version:

Petrov, D., Melnik, S., \& Hämäläinen, T. (2016). Distributed GNSS-Based Time Synchronization and Applications. In ICUMT 2016 : Proceedings of the 8th International Congress on Ultra Modern Telecommunications and Control Systems and Workshops (pp. 130-134). Institute of Electrical and Electronic Engineers. International Conference on Ultra Modern Telecommunications \& workshops. https://doi.org/10.1109/ICUMT.2016.7765345

All material supplied via JYX is protected by copyright and other intellectual property rights, and duplication or sale of all or part of any of the repository collections is not permitted, except that material may be duplicated by you for your research use or educational purposes in electronic or print form. You must obtain permission for any other use. Electronic or print copies may not be offered, whether for sale or otherwise to anyone who is not an authorised user. 


\title{
Distributed GNSS-Based Time Synchronization and Applications
}

\author{
Dmitry Petrov*, Sergei Melnik ${ }^{\dagger}$, Timo Hämäläinen ${ }^{\ddagger}$ \\ *Magister Solutions Ltd., Jyväskylä, Finland, dmitry.petrov@magister.fi \\ †Central Scientific Research Institute of Communication, Moscow, Russia, svmelnik@mail.ru \\ ${ }^{\ddagger}$ Faculty of Information Technology, University of Jyväskylä, Finland, timo.hamalainen@jyu.fi
}

\begin{abstract}
The paper deals with synchronization standards, applications and challenges in the modern packet switched telecommunication networks. In recent years, the number of time-dependent services and systems only increases with the development of such areas as Internet of Things (IoT), industrial Internet, remote automation and robotics, precise positioning etc. In the paper, these emerging applications are discussed. An alternative way to the traditional centralized synchronization infrastructure is proposed. Compact and low-priced devices equipped with a Global Navigation Satellite System (GNSS) receiver and a reliable internal clock can be installed specifically in the places where they are needed without additional intelligence in the network itself. Thus, distributed synchronization approach brings together the providers of time-critical information and sources of precise timing.
\end{abstract}

Index Terms-Synchronization, IoT, Industrial Internet, Timestamp, GNSS, NTP, PTP

\section{INTRODUCTION}

Many services running on modern digital telecommunications networks require accurate synchronization for correct operation. In general, synchronization can be defined in several different ways: firstly, it is frequency synchronization when different clocks come to the same state in the equal time intervals. One example of frequency synchronization source is Pulse Per Second (PPS) output. Secondly, certain systems may use multiple signals of different frequencies with a closely controlled phase relationship. These signals are in phase sync if they have a rising edge occurring at the same time with the lowest frequency signal [1]. Finally, if the explicit knowledge of time (i.e. timestamp) is required then time synchronization is considered. By time synchronism we will understand the identity of readings of different clocks in the same moment of time.

Coordinated Universal Time (UTC) is the common basis for the civil time used across the world. Timing centers have agreed to keep their time scales closely synchronized or coordinated. Two principal components are used to determine UTC: International Atomic Time (TAI) is the time scale that combines the output of highly precise atomic clocks worldwide; Universal Time (UT1) is so-called astronomic or solar time due to the rotation of the Earth. TAI is coordinated with UT1 in order to keep actual the length of a day on the Earth. In comparison to Greenwich Mean Time (GMT), which is only a name of the time zone, UTC is a time standard that
TABLE I

PRECISION LEVEL OF DIFFERENT CLOCKS [3]

\begin{tabular}{|c|c|}
\hline Type & Precision (seconds) \\
\hline \hline Cesium & From $10^{-11}$ up to $10^{-13}$ \\
\hline Hydrogen & From $10^{-11}$ up to $10^{-13}$ \\
\hline GPS & Around $10^{-12}$ \\
\hline Rubidium & From $10^{-9}$ up to $10^{-10}$ \\
\hline Crystal & From $10^{-5}$ up to $10^{-9}$ \\
\hline
\end{tabular}

is the basis for civil time and time zones worldwide [2]. That is why by correct time reference we will understand UTC.

Timekeeping of highest precision is provided by such devices as atomic clocks, for example, cesium (see Table I). However, the price level of such reference clocks is not affordable for common consumers and in many cases provided precision is redundant. On the other hand, even simplest sensors may possess time generators. The problem is that they often cannot provide necessary time stability in the long run because of the time drift. Thus, it is needed to synchronize these clocks with more precise sources. Next question is what device to select for this kind of synchronization? The obvious choice might be a common computer or a gateway. Such solution may be sufficient in some cases but is unacceptable in industrial applications. Firstly, the clock interval for most x 86 uniprocessors is about $10 \mathrm{~ms}$, and for most x 86 and x64 multiprocessors, it is about $15 \mathrm{~ms}$ [4]. Secondly, commuter's clock also requires synchronization to the external and more reliable source through the Internet. This connection typically goes through the network of Internet Service Provider (ISP), which is out of control for the consumer. Finally, in certain cases, legal time is required from a verified measuring device. Hence, reliable synchronization mechanism shall be provided.

In the modern and next generation communication networks in addition to the frequency synchronization requirements, there is a need to provide common time reference based on the UTC. There are at least the following reasons for that:

1) transition of transport networks from Time Division Multiplexing (TDD) systems such as Synchronous/Plesiochronous Digital Hierarchy (SDH, PDH) to the technologies based on packet switching (carrier Ethernet, metro Ethernet, Generalized Multi-Protocol Label Switching (GMPLS), etc.); 
2) development of content-oriented distributed services, indulging cloud, virtual, IoT systems;

3) development of new generations of mobile networks with advanced coordination, mobility, positioning features, etc.;

4) necessity to use timestamps in management, monitoring, billing systems including Quality of Service (QoS) guarantee and control;

5) implementation of new Information Technologies (IT) in the industries with high-reliability requirements.

For example, along with Voice over Internet Protocol (VoIP) transmission, special registration files are created in servers and routers. Each record in the registration file has a timestamp in order to keep the time of the events and their sequence. Files on the servers contain information from different sources. Thus, it is important that timestamps do not have mismatch over $1 \mathrm{~ms}$ according to the International Telecommunication Union (ITU-T) recommendation Y.1543 [5]. Otherwise, it is impossible to support guaranteed QoS in the network with a large number of geographically distributed entities.

Ensuring of required time difference between operator's network and UTC is also important for the IPTV services with Digital Rights Management (DRM). The license to view certain content is connected to the timestamp with the precision of $1 \mathrm{~ms}$. DRM systems are equipped with time servers of required precision and stability. However, it is not enough because their time scale should have a minimal mismatch with the equipment in the operators access network (Digital Subscriber Line Access Multiplexer (DSLAM), Gigabit-capable Passive Optical Networks (GPON), etc.) and also with the end users devices.

Time synchronization has high importance for the joint work of several telecommunication operators in packet switched networks. In this case, the guarantee of information security has special role essentially related to the time scale. Without unified time in operators network, it is impossible to provide even basic level of information security. Many methods of attacks are based on the modification of timestamps received from the public time servers.

It can be concluded that there is an obvious need to ensure synchronism of the network. Accordingly, the number of international standards related to network synchronization have been developed:

- 1PPS or Pulse Per Minute (PPM) sources can provide the highest accuracy of frequency synchronization but no extra information is transmitted.

- Network synchronization protocols:

The Network Time Synchronization (NTP) is one of the most commonly used network protocol for clock synchronization in large-scale packet-switched networks. It has undergone several releases since the year 1985. NTPv3 was specified in the Internet Engineering Task Force (IETF) Request for Comments (RFC)-1305 [6] and the latest version NTPv4 was proposed in RFC-5905 [7].

The Precision Time Protocol (PTP) defined in IEEE 1588 group of standards was developed to provide hardware-level time accuracy using a standard network connection by adding dedicated timing hardware to each port in a data network (e.g. Ethernet) [8]. It achieves clock accuracy in the sub-microsecond range in the applications where NTP or GNSS-based solution are inefficient or cannot be used.

- The Inter Range Instrumentation Group (IRIG) codes are used to efficiently interface the timing system output with the user system. In particular, atomic frequency standards and General Positioning System (GPS) receivers designed for precision timing are often equipped with an IRIG output [9].

- The IEEE 1344 extension to IRIG-B time codes later superseded by IEEE C37.118 defines synchronized phasor measurement for power systems [10]. In particular, it defines the use of IEEE 1588 PTP in power system applications.

- ITU-T recommendation G.8265 describes the deployment of IEEE 1588 standard in telecommunication networks for supporting the required timing precision and transfer of frequency [11].

- The IEEE 802.1 group of standards is developed by Time-Sensitive Networking (TSN) task group for data transmission over Ethernet networks. In particular, IEEE 802.1AS is the standard for transport of precise timing and sync in bridged Local Area Networks (LANs), e.g. audio/video bridging networks, data center bridging and other audio/video applications that need synchronization [12].

- The Synchronous Ethernet (SyncE) group of standards (G.8261, G.8262, and G.8264) developed by ITU-T in cooperation with IEEE specifies the architecture, clocks and messaging for transmitting synchronization signals over the Ethernet physical layer. Among the applications are cellular networks, access technologies such as Ethernet Passive Optical Network (EPON), and such services as IPTV or VoIP.

However, most of the time synchronization architectures still assume centralized or hierarchical time distribution. This approach will be discussed in more details in the next section, where distributed approach will be introduced as well. In Section III, traditional and emerging time-critical applications are presented and discussed. Finally, Section IV concludes the paper.

\section{TIME SYNCHRONIZATION ARCHITECTURE BASED ON GNSS}

In various applications, it is necessary to ensure a reliable and validated source(s) of synchronism. Satellite positioning systems such ass GPS, GLONASS and in perspective COMPASS/BeiDou and GALILEO provide an attractive opportunity for that purpose. The advantage of timing information provided by the Global Navigation Satellite Systems (GNSS) is based on following principle factors: firstly, satellites are equipped with atomic clocks of the highest precision and longterm stability because certain atoms (for example, Cesium- 


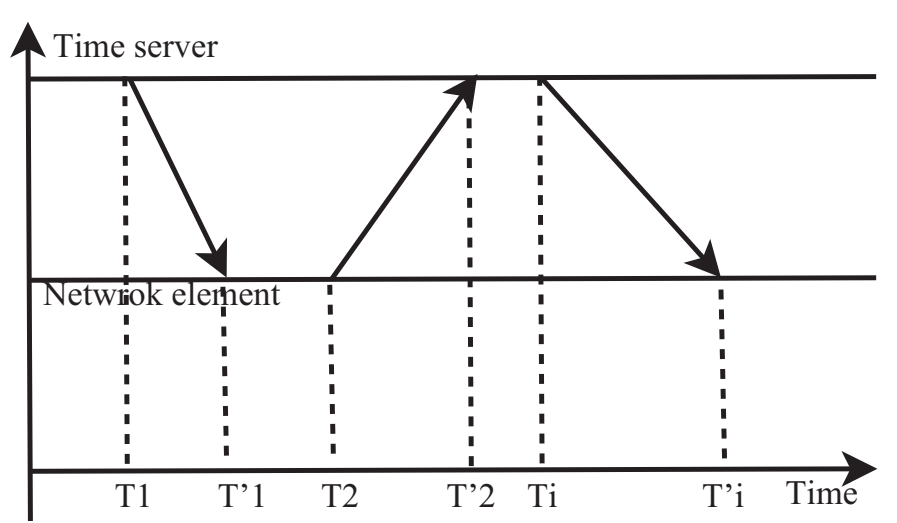

Fig. 1. Time synchronization and delay estimation.

133) resonate at extremely consistent frequencies. Secondly, on the assumption that a sufficient number of satellites is visible, it is possible to synchronize to these atomic clocks wirelessly from practically any point on the surface of the Earth. It means that GNSS-based time server is synchronous (with certain controllable error) directly to the atomic reference.

GPS signal is based on code-division-multiple-access (CDMA) and its reception includes time synchronization as one of the core components. GPS signal is pseudo-random phase-modulated sequence continuously transmitted at the high chip rate. Thus, the time difference between the clocks is defined by the autocorrelation of received satellite-specific sequence known at the receiver [13]. Time synchronization with GLONASS is more complicated. GLONASS-M satellites transmit the Frequency Domain Multiple Access (FDMA) navigation signals and time correction action is performed once per second. However, the situation should change when GLONASS-K is fully deployed with new CDMA signals in a separate frequency band added to the existing FDMA signals [14].

Utilization of only one GNSS system for synchronization have certain drawbacks:

- A receiving antenna requires free line-of-sight to the satellites, complicating installation. The trend is that the density of equipment deployment is only growing and receiver location optimization with respect to GNSS reception, therefore, becomes increasingly difficult. Furthermore, deployment of indoor devices will require an installation of an outdoor antenna, complicating the installation and increasing the cost.

- Any GNSS system may be switched off due to an outage or in a case of extraordinary military activities. Such accidents have already happened in the past for GPS and GLONASS.

Therefore, joint usage of all available GNSS systems together with stable internal time generator in a server provides much higher reliability. However, even if we already possess such a device it is still necessary to deliver synchronization signal or timestamps to the consumers with minimal errors.
In particular, packet-switched networks possess Propagation Dela Variation (PDV) [15]. Each transit router or switch adds a certain random contribution to the propagation delay of the packet. PDV depends on the traffic intensity, controller realization, temperature, quality of connectors and other parameters. Correct time recovery in the slave clock becomes a challenge. Network protocols have special algorithms to estimate time offset and cope with the delays [16]. As it is shown in Figure 1, measured time difference between the clocks of a time server and a network element $T_{1}^{\prime}-T_{1}=\theta+\delta$ includes $\delta$ - the actual transmit time and $\theta$ - the offset between the clocks. In the assumption of symmetrical channel, $T_{2}^{\prime}-T_{2}=-\theta+\delta$. Thus, from these two measurements the offset can be calculated:

$$
\theta=\frac{\left(T_{1}^{\prime}-T_{1}-T_{2}^{\prime}+T_{2}\right)}{2} .
$$

Measurement process in periodical so that estimated delays and offsets are filtered in order to remove statistical errors. However, non-symmetry of the channel is still a challenge especially at high loads.

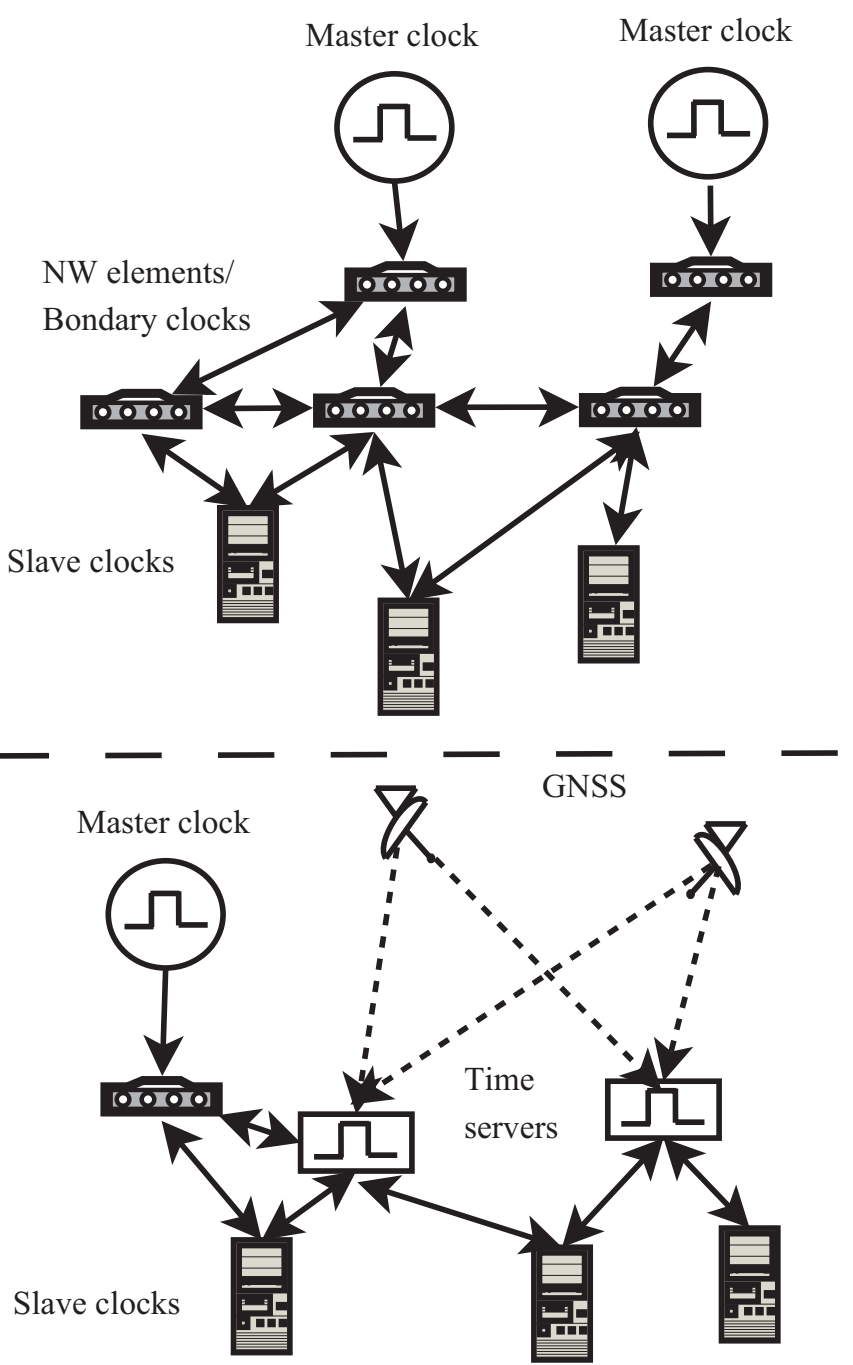

Fig. 2. Centralized/hierarchical (upper part) and distributed (lower part) time synchronization architectures. 
Moreover, in the ordinary networks with IEEE 1588 incompatible switches, synchronization messages are just transparently forwarded without a direct account of possible delays. Hence, achieved precision of NTP clients can be considerably reduced and highly dependent on network conditions. This issue can be partly solved with the use of IEEE 1588 compatible Boundary Clocks (BC) installed in the network elements. PDV in the slave clocks is minimized because BCs actually recover and regenerate synchronization signals (see the upper part of the Figure 2). The drawback of this approach is in increased implementation complexity and higher costs of the network infrastructure.

In the traditional hierarchical synchronization framework, the time reference is propagated from the very precise master or grandmaster clocks in several hops (stratums) to the destination devices. Basically, the more steps on the way of a packet with the timestamp the lower is the final precision. Therefore, the alternative solution proposed in this paper is to build infrastructure in such a way that time servers are placed as close as possible to the slave clocks. Such distributed architecture is presented on the lower part of the Figure 2 and is based on reasonably-prices compact devices possessing precise enough internal time generator that are synchronized to GNSS. Moreover, compact time servers can still benefit from network synchronization with the use of sync input. Finally, even if there are already existing time distribution mechanisms in the network (e.g. through the ISP), these devices can add necessary redundancy for the time-critical application.

Internal clocks of commercially available devices include double insulated quartz crystal and can possess thermal instability up to $\pm 5 \times 10^{-9} \mathrm{~s}$ and long-term instability of $\pm 5 \times 10^{-8}$ s per year. Hence, the achievable error limit of NTP synchronization over Ethernet port is on the level of $\pm 100 \mu \mathrm{s}$. Precision of 1PPS output can be definitely higher, i.e. an order of $\pm 1 \mu \mathrm{s}$ [17].

\section{ApPLiCATIONS}

There are various applications where synchronization is essential. Firstly, we present several traditional areas where it is already in use, as follows: Power lines and grids. Since all processes and events in a facility like a power substation are controlled from one singular central point, the absolute accuracy of the station's system time is not of high importance. However, as soon as time synchronized switching events involving more than one substation have to be performed the absolute accuracy of each stations time reference gains significant importance. Nowadays, a lot of measurement and control data in the power grid needs an absolute accuracy from $1 \mathrm{~ms}$ and up to $1 \mu \mathrm{s}$ or better. The power grids are complex and distributed interconnected system. Understanding, and possibly controlling, interactions of its elements require means to compare what is happening at one place and time with that happening at other places and times [18]. The following concrete processes need synchronization:

- event reconstruction on power substation level;

- synchrophasors measurements;
- system time and frequency deviation control;

- multirate billing;

- time tagging in measurement and fault detection systems, etc.

Telecommunications. As it was already discussed above, packet-switched networks are now widely deployed and should, therefore, be able to support all types of network applications. They are replacing the circuit switched synchronous SDH/SONET networks, that inherited mechanisms for accurate frequency and time references. Still many of the feature in modern and future wireless networks need synchronization, including but not limited to

- time and frequency references to mobile base station and correct generation of the signals on the radio interface and handover procedures;

- advanced features like Multicast/Broadcast Single Frequency networks (MBSFN), Cooperative Multipoint (CoMP), IntraCell Interference Coordination (ICIC), etc.;

- guarantee of QoS over transport networks (GPON optical transport, for example) and mobile backhaul, especially at high loads;

- precision of billing, etc.

Other areas that include automation of distributed systems, such as stock trading, transportation, cloud computing, oil/gas pumping etc.

Additionally, there are emerging areas of IT that can benefit specifically from the distributed synchronization infrastructure presented in the previous section.

Io $T$ in consumer and industrial applications. For industrial Internet, the information collected from different types of sensors can play a critical role in manufacturing and business operations. Due to power and computational constraints in the sensors themselves but also in the gateways, only limited amount of analysis can be done on these devices. Information may be still accumulated in network elements for fast online analysis. Later on, the measurements are sent to the large data centers for subsequent off-line processing. In this respect, evolved capillary network concept proposed by Ericsson can be mentioned as an example of such networks [19].

TThe data coming from geographically distributed sources should be aligned in time. Data mining algorithms, often used nowadays for the analysis of big data, need the correct sequence of events. Therefore, a mismatch in measurements from a certain group of sensors may result in incorrect alarms and decisions, unnecessary expenses or even damage in the equipment. Distributed GNSS-based synchronization is a solution that can considerably reduce the number of such problems. Even in the event of connection breaks the highest precision of time reference can be guaranteed with the locally installed time server. The chance that the timestamps have any offset is minimized.

Precise positioning. Positioning has even higher role in emerging 5G networks than ever before. The localization accuracy is expected to be on the level of 1 meter or below. With the densification of wireless networks, mobile terminals (MTs) 
are continuously connected to the small cells even at rather high speeds (e.g. in the cars, buses, etc.). In such environments, traditional channel measurements are resource consuming and, thus, inefficient. The possibility to estimate and track as well as predict the device locations in the radio network is highly beneficial. Network-centric positioning is a perspective approach because energy-consuming processing can be take away from the MTs. Uplink pilots already periodically send by the MTs can be used by the base stations (BSs) in order to collect necessary information and define the coordinates. Time difference measurements (e.g. time of Arrival (ToA)) is a usual component of positioning algorithms. However, the ultra-dense small cells or clusters of those, installed for example on the lamp posts, cannot always be interconnected through the wired backhaul. Timing offsets in those may cause errors in ToA. As it is shown in [20], Root Mean Square Errors (RMSEs) of coordinates' estimates is considerably dependent on the synchronism of the access nodes. The time servers places in the direct proximity of small cell would reduce time offsets, hence, improving the precision of positioning.

In-build localization is still one of the challenging areas of modern technology. Ultra Wide Band (UWB) signals have great potential for the Real Time Location Systems (RTLS) because achievable precision may be on the level of centimeters. DecaWaves DW1000 is one of the first commercially available hardware platforms on the market based on the UWB technology. Positioning is based on the Time Difference of Arrival (TDOA) technique which crucially depends on the time offsets in anchor nodes responsible for the measurements. Even using temperature controlled crystal oscillators with a very tight frequency tolerance (1 PPM) the time in different anchors will rapidly drift apart. For example, a 1 PPM error results in a $1 \mathrm{~ns}$ error over a $1 \mathrm{~ms}$ interval. This is well outside the limit required for accurate RTLS. As it is discussed in [21], time synchronization can provide a solution for this problem.

Legal time. More and more consumer services that require legally confirmed time are provided over the Internet. For example, public tenders, auctions usually have strict deadlines after which no bids are accepted anymore. The correct timing can be guaranteed only by specially verified devices. GNSS-based time servers receiving the signal directly from internationally accepted references on the satellites can be the acceptable source of such a timestamp. Taking into account the compact size and affordable price level of such devices, they can be also used in video surveillance systems even at homes. Only is this case, the records can be provided as a valid evidence in court after an accident.

An important application of time servers can be found in traffic offense control. Note, that a car moving at $60 \mathrm{~km} / \mathrm{h}$ per hour covers 16.7 meters in 1 second and at a speed of $100 \mathrm{~km} / \mathrm{h}$ it is already around 30 meters in one second. Thus, recording of such offenses as running through the red light or crossing double solid lines requires the use of two cameras. These cameras should work synchronously with the offset, not more than $10^{-2}$ seconds. Such level of precision can be provided by the co-located compact time server.

\section{CONCLUSION}

The main target of this work is to demonstrate how new types of GNSS-based time servers can be used in distributed manner for time-critical applications. Propagation of synchronization and time references over packet-switch networks is still challenging. Therefore, placing the source of sync in the proximity of its consumers provides a solution that can mitigate potential timing errors. This approach is efficient both from the network architecture and expenses point of view. In the paper, the state-of-the-art in network synchronization is overviewed. Next, distributed architecture is proposed. Finally, a number of traditional and emerging applications that benefit from such approach are highlighted.

\section{REFERENCES}

[1] "Phase Synchronization with Multiple Devices and Frequencies", Texas Instruments Inc., App. Rep., AN-1864, 2013.

[2] "UTC - The World's Time Standard" [Online]. Available: http://www. timeanddate.com/time/aboututc.html

[3] "Ethernet Network Synchronization", ALBEDO Telecom, White Paper, 2013.

[4] M. E. Russinovich, D. A. Solomon, and A. Ionescu, Windows internals, Pearson Education, 2012.

[5] Measurements in IP networks for inter-domain performance assessment, Recommendation ITU-T Y.1543, 2008.

[6] D. L. Mills, Network Time Protocol (Version 3) Specification, Implementation and Analysis, Request for Comments: 1305, Internet Engineering Task Force (IETF), Mar, 1992.

[7] D. L. Mills, J. Martin, J. Burbank and W. Kasch, Network Time Protocol Version 4: Protocol and Algorithms Specification, Request for Comments: 1305, Internet Engineering Task Force (IETF), Jun, 2010.

[8] IEEE Standard for a Precision Clock Synchronization Protocol for Networked Measurement and Control Systems, IEEE standard 1588, 2008.

[9] IRIG Serial time code formats, IRIG standard 200-04, Sept, 2004.

[10] IEEE Standard for Synchrophasor Measurements for Power Systems, IEEE standard C37.118.1, 2013.

[11] Precision time protocol telecom profile for frequency synchronization, Recommendation ITU-T G.8265.1/Y.1365.1, Jul, 2014.

[12] G. Garner, Standard for Local and Metropolitan Area Networks Timing and Synchronization for Time-Sensitive Applications, IEEE draft standard 802.1AS, 2016.

[13] G. Blewitt, "Basics of the GPS Technique: Observation Equations", in Geodetic Applications of GPS, Swedish Land Survey, 1997.

[14] Y. Urlichich, et al., "GLONASS Developing Strategy", Proceedings of the 23rd International Technical Meeting of The Satellite Division of the Institute of Navigation (ION GNSS 2010), pp. 1566-1571, Portland, OR, Sept. 2010.

[15] A. Morton, B. Claise, Packet Delay Variation Applicability Statement, Request for Comments: 5481, Internet Engineering Task Force (IETF), Mar, 2009.

[16] T. Gotoh, K. Imamura and A. Kaneko, "Improvement of NTP time offset under the asymmetric network with double packets method," Precision Electromagnetic Measurements, 2002. Conference Digest 2002 Conference on, pp. 448-449, Ottawa, Ontario, Canada, 2002.

[17] "Universal compact synchronization device - NTP/PTP server, technical characteristics" [Online]. Available: http://komset.ru/index.php/ ntp-server-ukus-pi-02dm.

[18] B. Dickerson, "Time in the Power Industry: How and Why We Use It", Arbiter Systems, Inc. [Online]. Available: http://www.arbiter.com/files/ product-attachments/TimeInThePowerIndustry.pdf.

[19] J. Sachs et al., "Capillary networks - a smart way to get things connected", Ericsson review journal, Aug, 2014.

[20] M. Koivisto et al., "Joint Device Positioning and Clock Synchronization in 5G Ultra-Dense Networks" [Online]. Available: http://arxiv.org/pdf/ 1604.03322v1.pdf.

[21] "Wired Synchronization of Anchor Nodes in a TDOA Real Time Location System", Application note, APS007, DecaWave, 2014. 